\title{
Improved Handoff Efficiency with the help of Neighbour Graph using Carrier to Interference Ratio
}

\author{
Debabrata Sarddar \\ Department of Electronics and \\ Telecommunication Engg, Jadavpur \\ University, Kolkata - 700032
}

\author{
Shubhajeet Chatterjee \\ Department of Electronics and \\ Communication Engg, Institute of \\ Engg. \& Managment college, \\ saltlake, Kolkata-700091.
}

Arnab Raha

Department of Electronics and Telecommunication Engg, Jadavpur University, Kolkata - 700032

\author{
Mandovi Mukherjee \\ Department of Electronics and \\ Telecommunication Engg, Jadavpur \\ University, Kolkata - 700032
}

\author{
Pulak Mazumder \\ Department of Electronics and \\ Telecommunication Engg, Regent \\ Education \& Research Foundation, \\ Barracpore
}

\author{
Utpal Biswas \\ Department of Computer Science \\ and Engg, University of Kalyani, \\ Nadia, West Bengal, Pin- 741235.
}

\author{
Mrinal Kanti Naskar \\ Department of Electronics and \\ Telecommunication Engg, Jadavpur University, \\ Kolkata-700032
}

\begin{abstract}
Presently, IEEE 802.11 based wireless local area networks (WLAN) have been widely deployed for business and personal applications. The main issue regarding wireless network technology is handoff or hand over management. When a mobile station (MS) moves outside the range of its current access point (AP) it needs to perform a link layer handover. This causes data loss and interruption in communication. According to IEEE 802.11, link layer2 (L2) handoff is performed in three phases scanning, authentication and re-association. Scanning process causes $90 \%$ of the total handoff delay. Throughout the last few years, plenty of researches had been done to reduce the hand off delay by reducing the scanning delay. In this paper, we propose to reduce handoff latency for IEEE 802.11 wireless networks with Neighbor Graphs (NG) pre-scanning mechanisms. IEEE 802.11 uses 11 channels of which the channels 1,6 and 11 do not mutually overlap. So these channels are expected to have a lower carrier-to-interference ratio (CI) compared to other channels under the same base station, which increases the channel's availability during handoff. Based on the NG prescanning mechanism, when handoff criterions have been met, we design an algorithm to first scan the channels 1, 6 and 11, if present under the next Access Point (AP), to reduce the scanning delay. We also introduce pre-authentication mechanism, which will effectively reduce the message processing delay.
\end{abstract}

\section{Keywords}

IEEE 802.11, Handoff latency, GPS (Global Positioning System), Base Station (BS), Mobile Station (MS), Neighbor APs.

\section{INTRODUCTION}

Handoff has become an essential criterion in mobile communication system especially in urban areas, owing to the limited coverage area of Access Points (AP). Whenever a MS move from current AP to a new AP it requires handoff. For successful implementation of seamless Voice over IP communications, the handoff latency should not exceed $50 \mathrm{~ms}$. But measurements indicate MAC layer handoff latencies in the range of $400 \mathrm{~ms}$ which is completely unacceptable and thus must be reduced for wireless networking to fulfil its potential.

With the advent of real time applications, the latency and packet loss caused by mobility became an important issue in Mobile Networks. The most relevant topic of discussion is to reduce the IEEE 802.11 link-layer handoff latency. IEEE 802.11 MAC specification [1] defines two operation modes: ad hoc and infrastructure mode. In the ad hoc mode, two or more stations (STAs) recognize each other through beacons and hence establish a peer-to-peer relationship. In infrastructure mode, an AP provides network connectivity to its associated STAs to form a Basic Service Set (BSS). Multiple APs form an Extended Service Set (ESS) that constructs the same wireless networks.

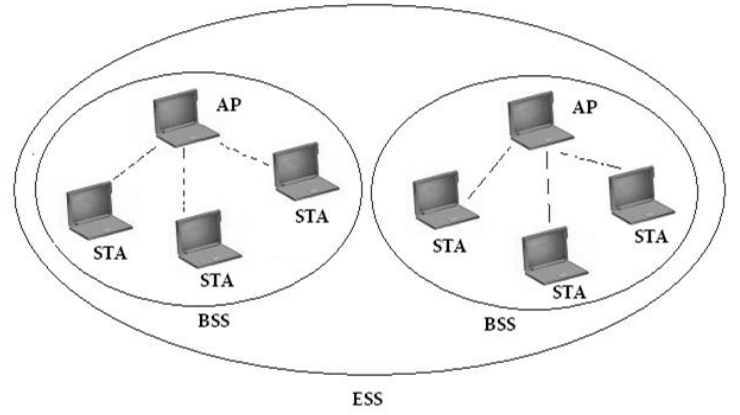

Figure 1. 


\subsection{Channel distribution}

IEEE $802.11 \mathrm{~b}$ and IEEE802.11g operates in the $2.4 \mathrm{GHz}$ ISM band and use 11 of the maximum 14 channels available and are hence compatible due to use of same frequency channels. The channels (numbered 1to14) are spaced by $5 \mathrm{MHz}$ with a bandwidth of $22 \mathrm{MHz}, 11 \mathrm{MHz}$ above and below the centre of the channel. In addition there is a guard band of $1 \mathrm{MHz}$ at the base to accommodate out-of-band emissions below $2.4 \mathrm{GHz}$. Thus a transmitter set at channel one transmits signal from $2.401 \mathrm{GHz}$ to $2.423 \mathrm{GHz}$ and so on to give the standard channel frequency distribution as shown in [Figure.2].

\section{Channels}

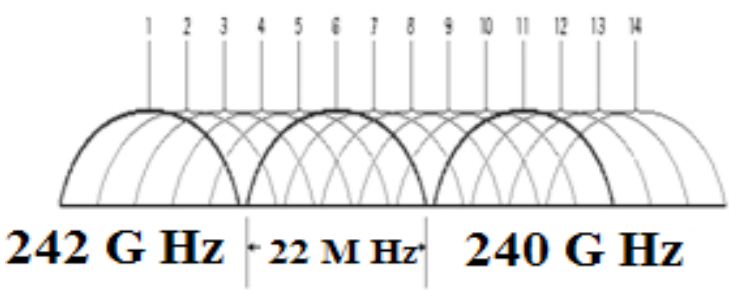

Figure.2 Channel Distribution

It should be noted that due to overlapping of frequencies there can be significant interference between adjacent APs. Thus, in a well configured network, most of the APs will operate on the non-overlapping channels numbered 1, 6 and 11 .

\subsection{Handoff}

When a MS moves out of reach of its current AP it must be reconnected to a new AP to continue its operation. The search for a new AP and subsequent registration under it constitute the handoff process which takes enough time (called handoff latency) to interfere with proper functioning of many applications.

Three strategies have been proposed to detect the need for hand off[2]:

1)mobile-controlled-handoff (MCHO):The mobile station(MS) continuously monitors the signals of the surrounding base stations(BS)and initiates the hand off process when some handoff criteria are met.

2)network-controlled-handoff (NCHO):The surrounding BSs measure the signal from the MS and the network initiates the handoff process when some handoff criteria are met.

3)mobile-assisted-handoff (MAHO):The network asks the MS to measure the signal from the surrounding BSs. The network make the handoff decision based on reports from the MS.

\section{Handoff can be of many types:}

Hard \& soft handoff: Originally hard handoff was used where a station must break connection with the old AP before joining the new AP thus resulting in large handoff delays. However, in soft handoff the old connection is maintained until a new one is established thus significantly reducing packet loss.

In NGWS(next generation wireless system), two types of handoff scenarios arise: horizontal handoff, vertical handoff[3][4].

$>$ Horizontal Handoff: When the handoff occurs between two BSs of the same system it is termed as horizontal handoff. It can be further classified into two:
- $\quad$ Link layer handoff : Horizontal handoff between two BSs that are under the same foreign agent(FA).

- Intra system handoff : Horizontal handoff between two BSs that belong to two different FAs and both FAs belong to the same gateway foreign agent (GFA) and hence to the same system.

Vertical Handoff : When the handoff occurs between two BSs that belong to two different GFAs and hence to two different systems it is termed as vertical handoff .

The handoff procedure consists of three logical phases where all communication between the mobile station undergoing handoff and the APs concerned is controlled by the use of IEEE802.11 management frames as shown below in [fig3].

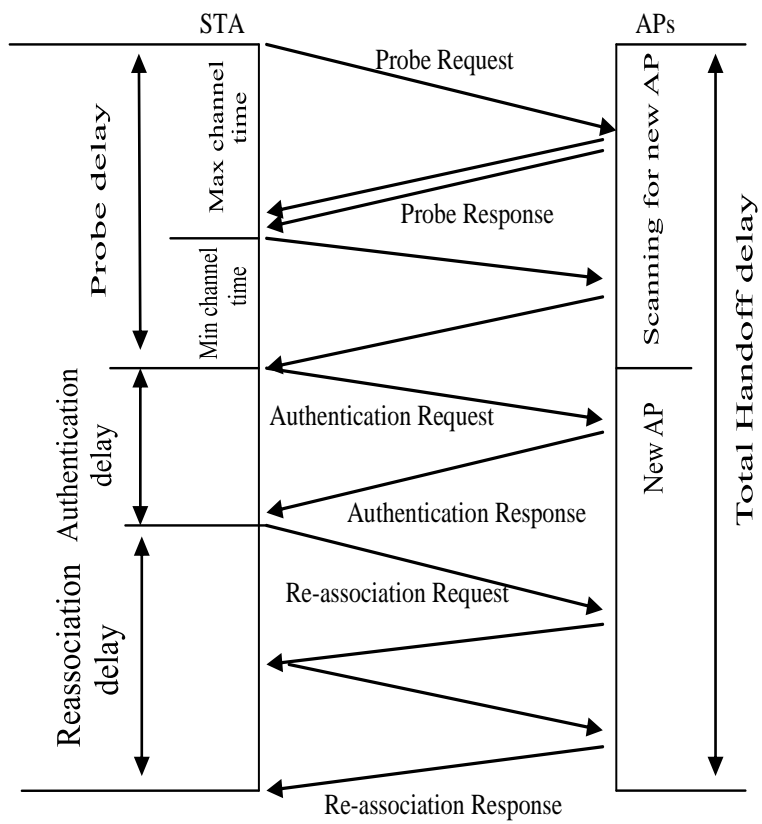

Figure 3. Handoff Process

Scanning: When a mobile station is moving away from its current AP, it initiates the handoff process when the received signal strength and the signal-to-noise-ratio have decreased significantly. The STA now begins MAC layer scanning to find new APs. It can either opt for a passive scan (where it listens for beacon frames periodically sent out by APs) or chose a faster active scanning mechanism wherein it regularly sends out probe request frames and waits for responses for $\mathrm{T}_{\mathrm{MIN}}$ (min Channel Time) and continues scanning until $\mathrm{T}_{\mathrm{MAX}}$ (max Channel Time) if at least one response has been heard within $\mathrm{T}_{\mathrm{MIN}}$. Thus, $\mathrm{n}^{*} \mathrm{~T}_{\mathrm{MIN}}$ $\leq$ time to scan $n$ channels $\leq n * T_{\text {MAX }}$. The information gathered is then processed so that the STA can decide which AP to join next. The total time required until this point constitutes $90 \%$ of the handoff delay.

Authentication: Authentication is necessary to associate the link with the new AP. Authentication must either immediately proceed to association or must immediately follow a channel scan cycle. In pre-authentication schemes, the MS authenticates 
with the new AP immediately after the scan cycle finishes. IEEE 802.11 defines two subtypes of authentication service: 'Open System' which is a null authentication algorithm and 'Shared Key' which is a four-way authentication mechanism. If Inter Access Point Protocol (IAPP) is used, only null authentication frames need to be exchanged in the re-authentication phase. Exchanging null authentication frames takes about 1-2 ms.

Re-Association: Re-association is a process for transferring associations from old AP to new one. Once the STA has been authenticated with the new AP, re-association can be started. Previous works has shown re-association delay to be around 1-2 $\mathrm{ms}$. The range of scanning delay is given by:-

$\mathrm{N} \times$ Tmin _ Tscan _ $\mathrm{N} \times \mathrm{Tmax}$

Where $\mathrm{N}$ is the total number of channels according to the spectrum released by a country, Tmin is Min Channel Time,Tscan is the total measured scanning delay, and Tmax is Max Channel Time. Here we focus on reducing the scanning delay by minimizing the total number of scans performed.

In section 2 we take you through the various works that have already been done to achieve this and in section 3 we explain our proposed method. This is followed by performance evaluation of our proposed technique using simulations in section 4 after which in section 5 we propose a few areas in which further improvement can be made. Finally, we provide an extensive list of references that has helped us tremendously in our work.

\section{RELATED WORKS}

A number of different schemes have been proposed to reduce handoff latency in IEEE 802.11 wireless LANs. IEEE 802.11 b based wireless and mobile networks [5], also called Wi-Fi commercially, are experiencing a very fast growth upsurge and are being widely deployed for providing variety of services as it is cheap, and allows anytime, anywhere access to network data. The new age applications require a seamless handover while the small coverage of individual APs has increased the number of handoffs taking place. Thus reducing the handoff latency has become a burning issue and much work has been done to achieve this. See [6] for an overall review of popular methods suggested.

Shin et al in [7] have introduced a selective scanning algorithm with the help of channel masking technique coupled with a caching mechanism to significantly reduce the handoff delay. However, it still scans excess APs even after the new AP may have already been found and thus leaves room for further improvements.

Handoff, an inherent problem with wireless networks, particularly real time applications, has not been well addressed in IEEE 802.11, which takes a hard handoff approach [8].

In [9] the authors have introduced a novel caching process using neighbor graphs by pre-scanning neighbor APs to collect their respective channel information. The concept of neighbor graphs can be utilized in different ways and have become very popular in this field. In [10] a pre-authentication mechanism is introduced to facilitate seamless handover. [11] is a novel approach towards reducing handover latency in AP dense networks.

Besides, much progress has been made in introducing GPS aided handoffs; vide [12] to [13]. To reduce handoff latency in wireless LAN using IAPP [14], an algorithm on context transfer mechanism using 'Neighbor Graph' (NG) [15] was suggested in [16]. However, IAPP was only reactive in nature and creates an additional delay in a handoff. One approach on Physical layer (PHY) is the method using two trans-receivers, where a wirelessMobile Station(MS) has two Wireless Network Interface Cards (WNICs) [17], one for keeping connection to current AP and the other for scanning channels to search for alternate APs [18].

Chung-Sheng $\mathrm{Li}$ et al. in [19] focused on neighbor graph caching mechanism for link layer handover. They use cache BSSIDs, SSIDs and channels of APs. They defined as,

$\mathrm{G}^{\prime}=\left(\mathrm{V}^{\prime}, \mathrm{E}\right)$

$\mathrm{V}^{\prime}=\left\{\mathrm{v}_{\mathrm{i}} \mathrm{v}_{\mathrm{i}}=\left(\mathrm{ap}_{\mathrm{i}}, \mathrm{BSSID}\right.\right.$, SSID and channel $\left.), \mathrm{v}_{\mathrm{i}} \in \mathrm{V}\right\}$,

$\mathrm{e}=\left(\mathrm{ap}_{\mathrm{i}}, \mathrm{ap}_{\mathrm{j}}\right)$,

$\mathrm{NC}\left(\mathrm{ap}_{\mathrm{i}}\right)=\left\{\mathrm{ap}_{\mathrm{ik}}: \mathrm{ap}_{\mathrm{ik}} \in \mathrm{V}^{\prime},\left(\mathrm{ap}_{\mathrm{i}}, \mathrm{ap}_{\mathrm{ik}}\right) \in \mathrm{E}\right\}$,

Where $G^{\prime}$ is the modified NGC and $V^{\prime}$ is the set containing APs with BSSIDs, SSIDs and channels of neighbor APs.

$E$ is the set of edges. $N C$ is the neighbor APs of an AP. By this process they significantly reduce the handoff delay. It saves time as NGC consists of APs, channels and additional information of BSSIDs and SSIDs.

In our work also we look at another such position dependant solution with a view to minimize overhead signalling problems. This is necessary since extensive pre-scanning is unacceptable in high traffic AP dense networks.

In [6], a new handover management technique has been proposed using neighbour graph.

In [7] the authors have introduced a novel caching process using neighbor graphs by pre-scanning neighbor APs to collect their respective channel information. The concept of neighbor graphs can be utilized in different ways and have become very popular in this field. In [8] a pre-authentication mechanism is introduced to facilitate seamless handover. [9] is a novel approach towards reducing handover latency in $\mathrm{AP}$ dense networks.

Besides, much progress has been made in introducing GPS aided handoffs; vide [10], [11], [12], [13], [14]. In our work also we look at another such position dependant solution with a view to minimize overhead signalling problems. This is necessary since extensive pre-scanning is unacceptable in high traffic AP dense networks.

\section{PROPOSED WORK}

The maximum range up to which the signal can be transmitted is determined by the height of the antenna and the power of the signal is inversely proportional to the square of the distance from the AP. But due to fading, the signal strength is never equally spread in all direction even for an omni-directional antenna. There are mainly two types of fading responsible for the uneven distribution of the signal strength from the AP. They are namely "fast fading" (fading due to scattering of the signal by object near transmitter) and "slow fading" (fading due to long term spatial and temporal variations). Ideally without fading, the cell's coverage area would be circular, but due to fading it becomes an undefined contour. Signal strength contours for two APs operating in ideal condition without fading is given in Figure 4 (a) and operating in real condition with fading is given in Figure 4 (b). Here we define the coverage area of each AP to be concentrated within a hexagon of certain edge length, which is the best approximation so far considering uneven distribution of signal.

The same frequency band or channel used in a cell can be reused by another cell as long as the cells are far apart and the signal strength does not interfere with each other (this mechanism is 
shown in Figure 5 where the three yellow marked cells can use the same frequency channel to avoid frequency interference). Thus frequency channels are allocated in such a way that the interference due to any two neighboring APs, i.e., the co-channel interference is minimum. But within a cell, the channels used may be mutually overlapped and this may lead to interference within the same AP. We cannot reduce both the types of interference simultaneously. But for optimizing it to a minimum value, the channel allocation protocol is designed in such a way so that at least one of channels 1,6 and 11 are made available to each AP. This is due to the fact that the channels 1, 6 and 11 are mutually non-overlapping. Due to increasing traffic, the need of greater frequency range is realized. Hence, by implementing the above procedure, we can thus use a maximum number of channels in one AP with at least one channel having minimum interference even in regions where signal strength is very low, thereby increasing the net signal to noise ratio (SNR).

Now handoff is primarily dependent upon signal strength received and the CI ratio. The term CI ratio is the ratio between the frequency band allocated to the MS and the interference associated with this frequency band. Thus as per our theory, channels 1, 6 and 11 are expected to have a greater CI ratio than the others. So, during handoff, if we scan channels 1, 6 and 11 , if present, first, then there will be a greater probability that the call is transferred to any of these channels of the next AP. So based on CI ratio, it is seen that scanning non over-lapping channels is better for reducing the latency due to scanning delay.

Second generation wireless systems and most of the research works follow Frequency Division Multiple Access (FDMA) or Time Division Multiple Access (TDMA) for multiple access of a single channel frequency band. In TDMA, one channel is used by several users, with AP assigning time slots for different users, and each user is served in a round-robin method. In FDMA, the allocated frequency band for one channel is subdivided into many sub-bands and each sub-band is allocated by the AP to each user. Thus, in FDMA, it may be seen that a particular subband is allocated to a user which falls between the interference zones of channels within the same AP. Thus, protocols using FDMA techniques have a certain probability that during handoff, even when the channel is free, the user is allocated such a sub-band within the above mentioned region. Thus, it will encounter a very low CI ratio and the ceases to operate on that channel and scans for the next channel. During scanning of the non over-lapping channels, this problem will not be faced. So our method works even better in cases where FDMA is used for multiple access of a single channel.

With these assumptions, we propose (i) a selective channel scan, as in [3], and (ii) pre-authentication scheme with the help of neighbor graph for reducing the total handoff delay.

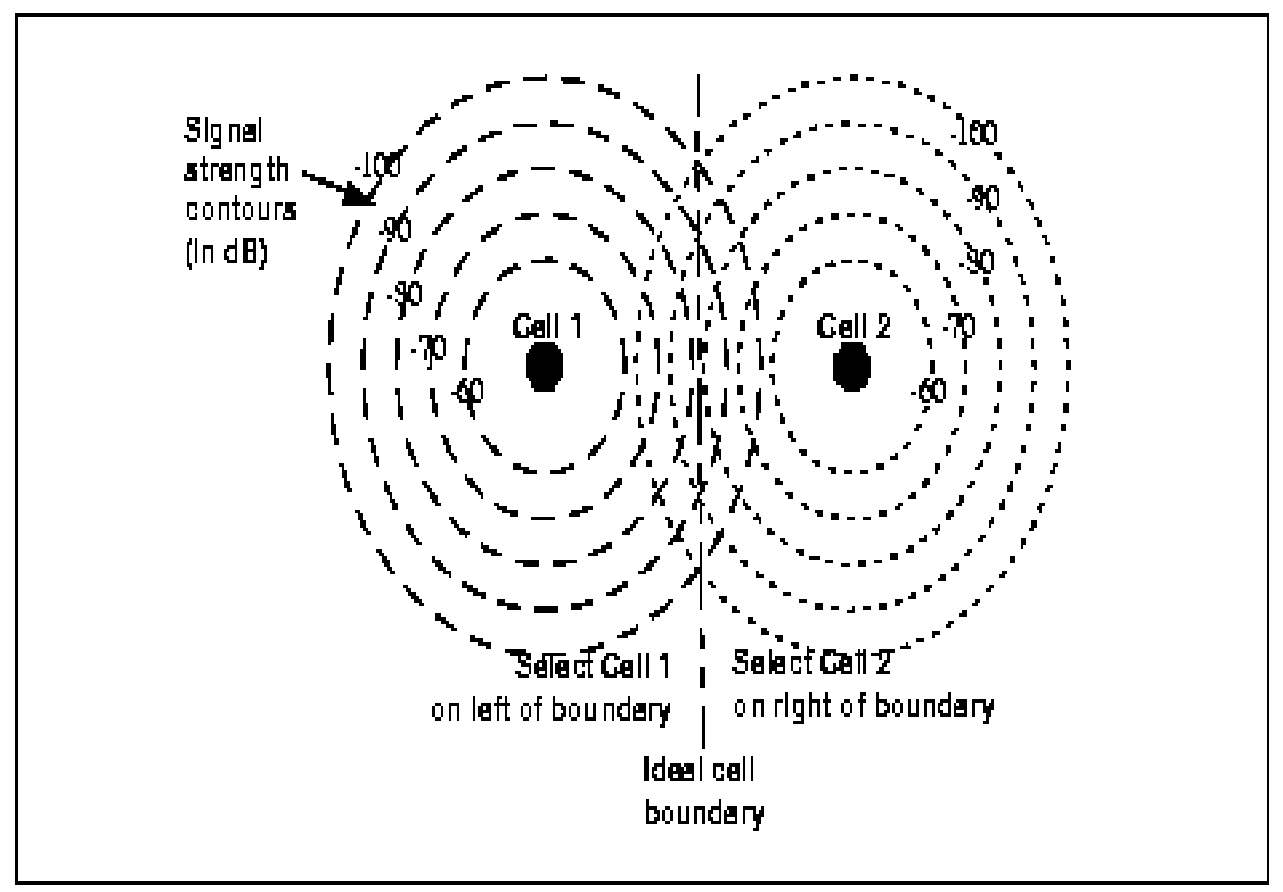

Figure 4 (a). It shows the distribution of signal strength of APs in ideal condition 


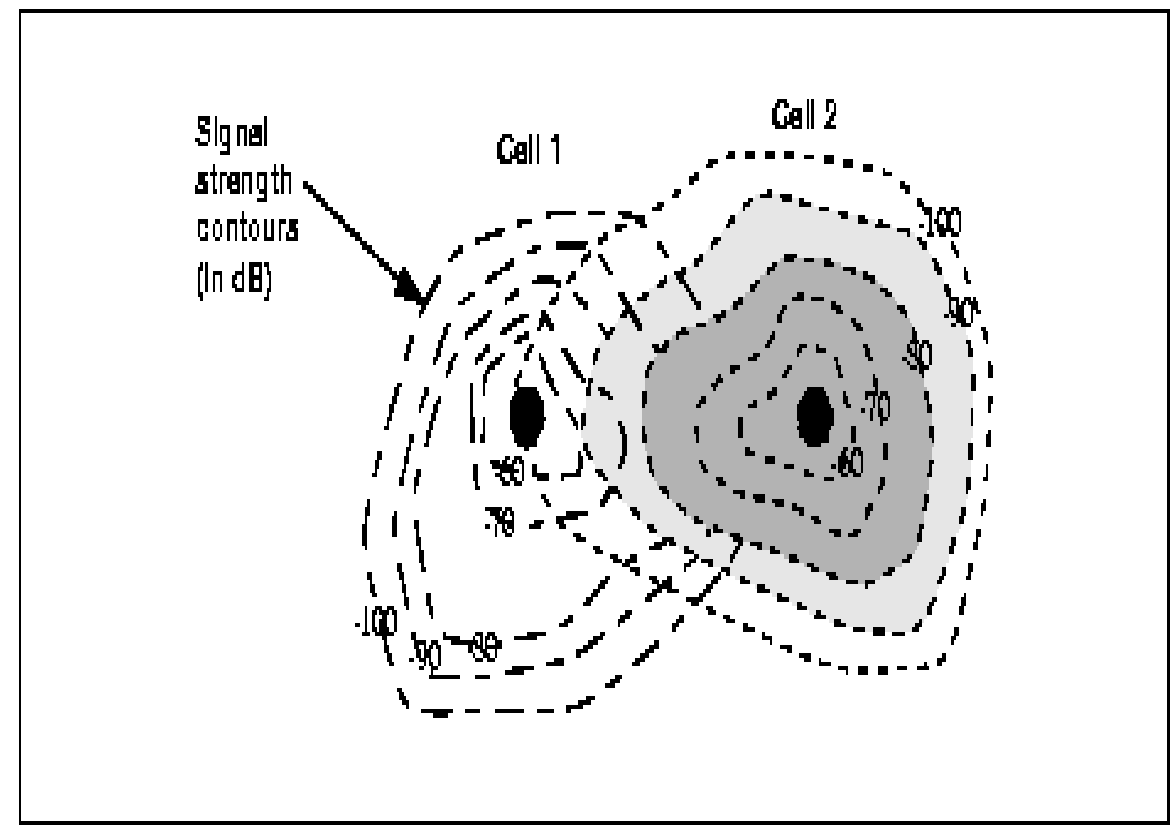

Figure 4 (b). It shows the distribution of signal strength of APs in real condition

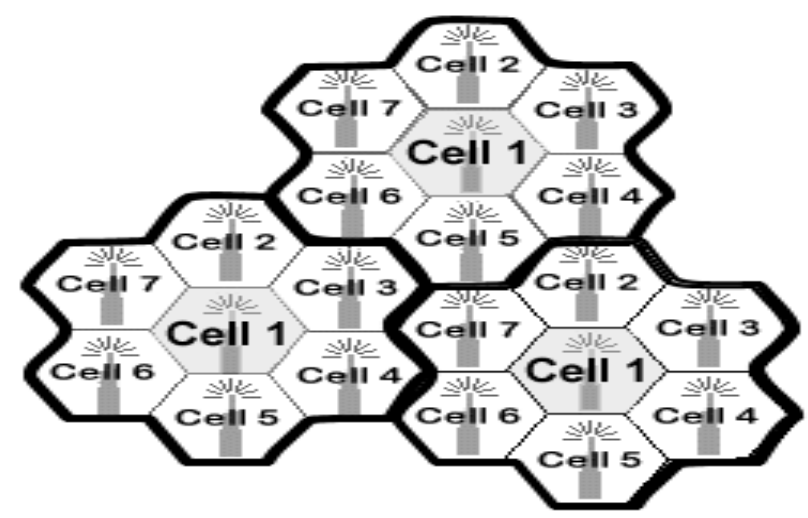

Figure 5. Diagram representing cells that can use the same frequency channels.

\subsection{Selective channel scanning}

As in [14], the MS downloads from the server the data which not only contains the neighbor of the AP on which it is presently operating, but also the channels used by the neighboring APs. However the MS must wait for "min channel time" or "max channel time" as the MS does not know how many APs would respond to the probe request. So here we use unicast instead of broadcast which selects the potential APs to which the call may be handed off and scans only the channels associated with those APs. Selective channel probing with the help of unicast instead of broadcast brilliantly reduces the handoff delay by a massive percentage when compared with selective scanning or basic active scanning. Moreover, it was also stated that the MS has to wait for only the 'round trip time' (rtt) for scanning each channel instead of the min channel time or the max channel time. We know that IEEE uses 11 out of the 14 possible channels, out of which 1, 6 and 11 are mutually non-overlapping. When the MS responds to handoff, according to the pre-scanning mechanism of NG, it first looks for the potential AP and then first scans the channels 1,6 and 11 if present. If this fails, it will start scanning the other channels. As proposed in [3], the expected scanning delay using selective scanning is

$$
\mathrm{t}=\mathrm{N}^{\prime} \times \tau+\alpha
$$

where ' $t$ ' is the scanning delay, $N$ ' is the number of channels scanned, ' $\tau$ ' is the round trip time and $\alpha$ is the message processing time. ' $\tau$ ' is the summation of the time taken for the Probe Request to be sent to the selected APs and for the Probe Response to be received, which, in our case, is nothing but the Min Channel Time, which has been estimated to be around 3-7 ms.

\subsection{Pre authentication}

To reduce the message processing delay, authentication is done during scanning phase. By this method, the authentication delay vanishes and the message processing delay, $\alpha$, is composed only of the re-association time. Thus the parameter ' $\alpha$ ' is reduced by at least half of its initial value and hence the net time delay, $t$, as proposed in [3], is greatly reduced. This can be implemented as proposed in [19] and [20].

Thus, the authentication time, which was very minute in proportion as compared to scanning phase delay of previous methods, would now command a greater percent of time delay, because, in our case, the scanning phase delay has been much reduced. However, by the process of pre-authentication, even this delay is nullified. So our method reduces the net handoff latency by a great extent as compared to the previous proposed methods. The experimental results are given in the forthcoming section. It gives a brief overview of the simulation process and the results obtained conform to our theory. 


\section{SIMULATION RESULTS}

For the simulation part, we used a 2D-plane with APs on centre of hexagons packed together. The heights of each antenna were considered to be the same and the topology distribution was also considered to be similar. We used hexagons for specifying the range of the APs as hexagons can fit side by side like honey combs and is mainly used by all research workers for this category of simulations. The frequency was allocated as per the protocols that are generally followed in frequency allocation in IEEE 802.11 standards. Calls originate on a memory less basis, that is, they follow Poisson Distribution function and use the channel within the AP on whose range the call is created. The channel allocated to it is determined by the AP. The channel allocation is considered to be static and FDMA was used for multiple accesses. The CI ratio was calculated for each channel within each AP which is an important parameter for our method. Moreover, separate CI ratios were calculated for all the sub bands when a single channel was used by multiple users. The sub bands' CI ratio takes into account the CI ratio of the channel on which the multiple access is carried on. Now, we considered various instances of time where there is a case where a randomly generated MS has a need for handoff. The different parameters, like the number of existing MSs in the two APs, the CI ratios etc. at that instance of time were taken into consideration

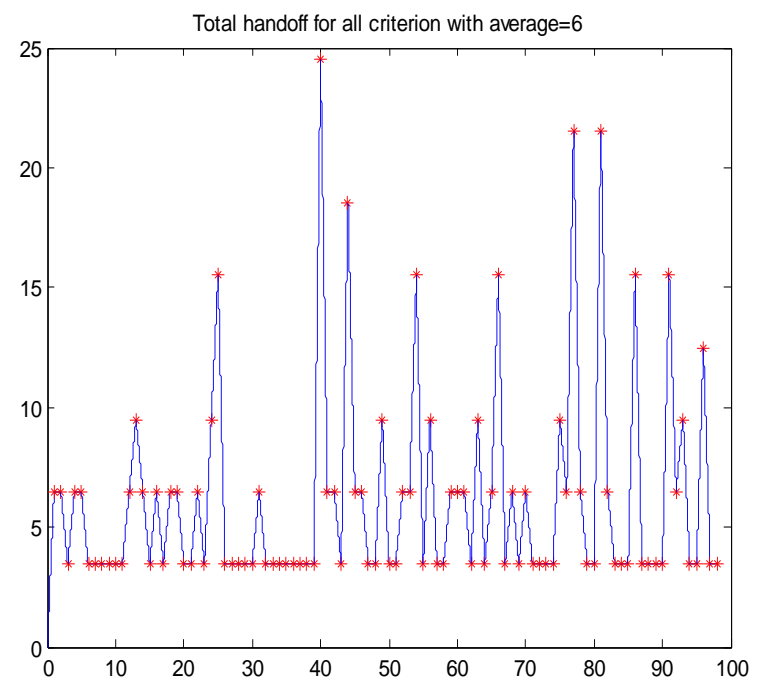

Figure 6. Graph showing the handoff delays at various instances

. Then we applied our method, i.e., the MS first looks for the potential AP and then first scans the channels 1,6 and 11 if present. If this fails, it will start scanning the other channels. We also neglect the authentication delay as pre-authentication was done during the scan phase. We considered the round trip time to be $3 \mathrm{~ms}$ and the message processing time which comprises of only the re-association time was neglected to carry out our calculations.

We made a sample run of our simulation and calculated the time required for handoff at regular intervals of time. We calculated 100 such instances and calculated the total time required for the handoff to take place. From a particular sample run we got the average time delay for all 100 instances as 6.1129 $\mathrm{ms}$. The graph of this simulation is plotted in Figure 6, which shows the various handoff delay times in the ' $\mathrm{Y}$ ' axis in milliseconds, for each instance, which is shown in the ' $X$ ' axis. The variation of results obtained from other simulation was negligible. So we can consider that our method reduces the net handoff delay to a minimum of around $6 \mathrm{~ms}$ in the best case which is much lower than the previous results.

\section{CONCLUSION}

In this paper, we claim our contributions as stated below:

We have discussed a pre-scanning procedure using channels 1,6 and 11 , which greatly reduces the scanning time. Here, we have set a best scenario time of about $6 \mathrm{~ms}$, which is an improvement on the best scenario time of $18 \mathrm{~ms}$ previously set .

We have used pre-authentication in which the STA authenticates the new AP just after the scanning phase, thus minimizing the message processing delay.

Our discussion is based on IEEE $802.11 \mathrm{~b}$ standard, even though the proposed set-up is also valid for IEEE 802.11g with minor adjustments. As is evident, the discovery phase is still the most time consuming phase of the handoff process. Future simulations may be done using different topologies with modifications regarding selective scanning and preauthentication using IAPP. Moreover, our method works best in networks where FDMA is used for multiple access. Though networks using TDMA has also reduced handoff latency by this method, but still in the best case scenario, the net time delay is a bit more than that of networks using FDMA. So, further research work can be done in this field.

We have considered link layer handoff delay only. For intrasystem handoff, a bit more time will be required due to increase in message processing delay. Though we have nullified the authentication delay, yet the re-association delay cannot be neglected while doing the simulations. But for this case, as the re-association delay is very small as compared to the handoff delay, so we can neglect it for our experimental results.

\section{REFERENCES}

[1] Hye -Soo Kim, Sang Hee Park, Chun-Su Park, Jae Won Kim and Sung-Jea Ko. "Selective Channel Scanning for Fast Handoff in Wireless LAN using Neighbor Graph", July 2004.

[2] Hongqiang Zhai, Xiang Chen, and Yuguang Fang. "How well can the IEEE 802.11 wireless lan support quality of service?" IEEE Transactions on Wireless Communications, 4(6):3084-3094, December 2005.

[3] Yi-Bing Lin Imrich Chalmatc, "Wireless and Mobile Network Architectures," pp. 17.

[4] AKYILDIZ, I. F., XIE, J., and MOHANTY, S., "A survey on mobility management in next generation all-IP based wireless systems," IEEE Wireless Communications, vol. 11, no. 4, pp. 16-28, 2004.

[5] STEMM, M. and KATZ, R. H., "Vertical handoffs in wireless overlay networks," ACM/Springer Journal of Mobile Networks and Applications(MONET), vol. 3, no. 4, pp. 335-350, 1998.

[6] Jaeyoung Choi Student Member, IEEE, Taekyoung Kwon \& Yanghee Choi, Senior Member IEEE, Sangheon Pack Member, , IEEE,Fast Handoff Support in IEEE 802.11 Wireless Networks. 
[7] Arunesh Mishra, Minho Shin \& William Arbaugh, An Empirical Analysis of the IEEE 802.11 MAC Layer Handoff Process. [Anshuman Singh Rawat \& Henning Schulzrinne Reducing MAC Layer Handoff Latency in IEEE 802.11 Wireless LANs.

[8] Sangho Shin, Andrea G. Forte, Context Caching using Neighbor Graphs for Fast Handoffs in a Wireless Network.

[9] S. Park and Y. Choi Pre-authenticated fast handoff in a public wireless LAN based on IEEE802.1x mode IFIP TC6 Personal Wireless Communications. Singapore, October 2002.

[10] Jin Teng, Changqing Xu, Weijia Jia, Dong Xuan, D-scan: Enabling Fast and Smooth Handoffs in AP-dense802.11 Wireless Networks.

[11] Chien-Chao Tseng, K-H Chi, M-D Hsieh \& H-H Chang, Location-based Fast Handoff for 802.11 Networks. IEEE COMMUNICATIONS LETTERS, VOL9, NO 4 April 2005.

[12] S.Kyriazakos, D. Drakoulis, G.Karetsos, Optimization of the Handover Algorithm based on the Position of the Mobile Terminals. Proceedings of Symposium on Communications and Vehicular Technology, October 2000 .

[13] In-Su Yoon, Sang-Hwa Chung, and Tae-Hoon Kim, A fast handover method for IEEE802.11 wireless networks using combined GPS and SNR

[14] J. Pesola \& S. Pokanen, Location-aided Handover in Heterogeneous Wireless Networks. in Proceedings of Mobile Location Workshop, May2003.

[15] Jan Eric Hakegard, Multi-Cell WLAN Coverage and Capacity.

[16] Ping-Jung Huang, Yu-Chee Tseng. "A Fast Handoff Mechanism for IEEE 802.11 and IAPP Networks".

[17] M.Ohta, "Smooth Handover over IEEE 802.11 Wireless LAN, "Internet Draft : draft-ohta- smooth- handoverwlan-00.txt, Jun. 2002.

[18] Yogesh Ashok Powar and Varsha Apte, "Improving the IEEE 802.11 MAC Layer Handoff Latency to Support Multimedia Traffic".

[19] Chung-Sheng Li et.al. 'A Neighbor Caching mechanism for Handoff in IEEE 802.11 Wireless Networks.' Springer 20 March 2008,DOI 10.1007/s11227-008-0175-3.

\section{AUTHORS PROFILE}

Debabrata Sarddar is currently pursuing his $\mathrm{PhD}$ at Jadavpur University. He completed his M.Tech in Computer Science \& Engineering from DAVV, Indore in 2006, and his B.Tech in Computer Science \& Engineering from Regional Engineering College, Durgapur in 2001. His research interest includes wireless and mobile system.

Shubhajeet Chatterjee is presently pursuing B.Tech Degree in Electronics and Communication Engg. at Institute of Engg. \& Managment College, under West Bengal University
Technology. His research interest includes wireless sensor networks and wireless communication systems.

Arnab Raha is presently pursuing B.E. (3rd Year) in Electronics and Telecommunication Engg. at Jadavpur University. His research interest includes wireless sensor networks, advanced embedded systems and wireless communication systems.

Mandovi Mukherjee is presently pursuing B.E in Electronics and Telecommunication Engg. at Jadavpur University. Her research interest includes wireless sensor networks and wireless communication systems.

Pulak Mazumder received his M.Tech in Electronics \& Telecommunication Engineering from WBUT, Kolkata in 2010, and his B.Tech(AMIETE) in Electronics \& Telecommunication Engineering from IETE, New Delhi in 2006. At present, he is an Asst. Professor in the Department of Electronics and Tele- Communication Engineering, Regent Education \& Research Foundation, Barracpore. He was earlier a lecturer in ECE dept. at Durgapur Institute of Advanced Technology, Durgapur and Calcutta Institue of Engineering and Mangement, Tollygung, Kolkata. He served Industry more than eight years as IT Network Infrastructure Management. His research interest includes wireless and mobile communication systems.

Utpal Biswas received his B.E, M.E and $\mathrm{PhD}$ degrees in Computer Science and Engineering from Jadavpur University, India in 1993, 2001 and 2008 respectively. He served as a faculty member in NIT, Durgapur, India in the department of Computer Science and Engineering from 1994 to 2001. Currently, he is working as an associate professor in the department of Computer Science and Engineering, University of Kalyani, West Bengal, India. He is a co-author of about 35 research articles in different journals, book chapters and conferences. His research interests include optical communication, ad-hoc and mobile communication, semantic web services, E- governance etc.

Mrinal Kanti Naskar received his B.Tech. (Hons) and M.Tech degrees from E\&ECE Department, IIT Kharagpur, India in 1987 and 1989 respectively and Ph.D. from Jadavpur University, India in 2006.. He served as a faculty member in NIT, Jamshedpur and NIT, Durgapur during 1991-1996 and 19961999 respectively. Currently, he is a professor in the Department of Electronics and Tele-Communication Engineering, Jadavpur University, Kolkata, India where he is in charge of the Advanced Digital and Embedded Systems Lab. His research interests include ad-hoc networks, optical networks, wireless sensor networks, wireless and mobile networks and embedded systems. He is an author/co-author of the several published/accepted articles in WDM optical networking field that include "Adaptive Dynamic Wavelength Routing for WDM Optical Networks" [WOCN,2006], "A Heuristic Solution to SADM minimization for Static Traffic Grooming in WDM unidirectional Ring Networks" [Photonic Network Communication, 2006], "Genetic Evolutionary Approach for Static Traffic Grooming to SONET over WDM Optical Networks" [Computer Communication, Elsevier, 2007], and "Genetic Evolutionary Algorithm for Optimal Allocation of Wavelength Converters in WDM Optical Networks" [Photonic Network Communications, 2008]. 